\title{
Effects of Therapeutic Education on Adherence to Therapeutic Prescriptions in ADPKD Patients: A Quasi -Experimental Study Protocol
}

\section{Brioni E*}

San Raffaele Hospital, Italy

*Corresponding author: Elena Brioni, San Raffaele Hospital, Via Olgettina 60, Italy, Tel: 3461608134; Email: brioni.elena@hsr.it

\section{Research Article \\ Volume 2 Issue 6}

Received Date: November 08, 2018

Published Date: December 05, 2018

DOI: $10.23880 /$ nhij-16000164

\begin{abstract}
Background: Autosomal dominant polycystic kidney disease (ADPKD) is the most common inherited renal disease and affects less than 5 people in 10000. There are many effective treatments, including blood pressure management, physical activity, low sodium diet and hydration. Nursing therapeutic education is part of the care of the patient affected by ADKPD and includes knowledge and management of the co-morbidities, level of compliance of the pharmacological therapy, with a focus on anti-hypertensive therapy and Tolvaptan, compliance with dietetic advices and lifestyle adjustments.

Methods: A sample of 50 patients affected by ADPKD will receive an intervention that consists of receiving education about pharmacological therapy, diet and lifestyle. The nurse will use standard educational procedures to inform the patients about the importance of compliance to pharmacological therapy, low sodium diet and moderate physical activity.

Results: Therapeutic education in patients affected by ADPKD with chronic kidney disease (CKD) can have a positive impact on patient's health by improving compliance with pharmacological therapy, diet and lifestyle.

Conclusions: Therapeutic education improves the patient's knowledge of the disease, treatments and correct behaviors in order to promote an independent management of the disease. The patient would be empowered to modify wrong behaviors and obtain a balance between his and the disease's needs and therefore improving compliance with treatments and improving quality of life.
\end{abstract}

Keywords: Therapeutic Education; Adherence; Therapeutic Prescriptions; Polycystic Kidney Disease

Abbreviations: ADPKD: Autosomal Dominant Polycystic Kidney Disease; CKD: Chronic Kidney Disease; CRF: Case Report Form; PACIC: Patient Assessment of Chronic Illness Care; IPAQ: International Physical Activity
Questionnaire; SMAQ: Simplified Medication Adherence Questionnaire; MHLC: Multidimensional Health Locus of Control Scale; BIPQ: Illness Perception Questionnaire Revised Brief; PAM: Patient Activation Measurement; 


\section{Nursing \& Healthcare International Journal}

KDQOL-SF: Kidney Disease Quality of Life - Short Form; CRF: Case Report Form; IHLC: Health Locus of Control; PHI: Physical Health Index; MHI: Mental Health Index.

\section{Introduction}

\section{Background}

Autosomal dominant polycystic kidney disease (ADPKD) is the most common inherited renal disease and affects less than 5 people in 10000 [1].

The disease is characterized by a prolonged subclinical course of gradual renal cyst expansion, resulting in massively enlarged kidney, reduced renal functionality and the progression of the renal disease to the final stage. The initial signs and symptoms are hematuria, hypertension, pain and fatigue [2].

Many treatments are considered effective; amongst them are blood pressure management, physical activity, low sodium diet and hydration. Salt consumption is a strong independent predictive factor for cardiovascular and renal adverse events in patients with chronic kidney disease (CKD). By reducing and maintaining a low consumption of salt, it is possible to maximize the effect of ACE inhibitors on slowing the progression of CKD [3].

The understanding of the importance of these treatments and the compliance to the recommendations are variable amongst patients [4]. To improve patient's compliance is necessary to provide a better education and highlight positive messages about diet and lifestyle [5].

ADPKD has been neglected for a long time due to lack of treatments but now there is a new interesting treatment available: Tolvaptan has shown clinical effectiveness on the disease progression in two different studies (patients with mild renal failure and patients affected by ADPKD with advanced renal failure). Despite the scientific evidence, hepatic toxicity (in about 4\% of the subjects that received the drug) and the important diuretic effect suggest caution in using this new treatment [6].

Therapeutic education is part of patient's care and treatment. This approach represents an essential strategy in order to face the current healthcare scenario where there is a progressive increase of people affected by chronic diseases. It is in this epidemiological scenario that there are opportunities in education because it considers the individual the main responsible for their own health and prompts the prevention of complications due to the disease. Education has the autonomy of the patient and the careers as main objective [7].

This article aims to analyse the effect of therapeutic education on the patient affected with ADPKD, their knowledge about co-morbidities, the level of compliance to pharmacological therapy (anti-hypertensive therapy and Tolvaptan), their compliance to dietetic and lifestyle recommendations as part of a nursing led education process in a specialistic clinic.

\section{Framework}

Current evidence indicates that people with chronic diseases have better outcomes when an effective treatment is delivered as part of an integrated framework that includes self-management support and regular follow-up [8]. If the patients have more knowledge about the disease, are more involved in the care process, and made feel more responsible, they have a more effective relationship with the healthcare professionals and put more commitment in obtaining positive outcomes for their health status [9].

Therapeutic education is part of the secondary prevention phase (if a risk factor is present it aims to delay the disease as possible) and tertiary prevention phase (with the presence of the disease it aims to delay the onset of complications). It consist in a real "transfer", planned and organized, of competencies from the healthcare professionals to the patient as part of a perspective where the dependency of the patient is set apart progressively by the responsibility felt by the patient and the partnership with the healthcare team [10].

Recently, the concept of "Patient Engagement" has gained attention by healthcare institutions; in other words, it can be called "patient's active involvement". It is identified as condition "sine qua non" by international scientific evidence for healthcare innovation. Nonetheless, in the last decades there has been a deep turning of healthcare models towards an increased promotion of the role of the patient, seen as an active subject and "expert" within the clinical process [11].

The involvement of the patient affected by a chronic disease is regarded as an important factor towards a good compliance to the therapeutic treatments. Therapeutic adherence means the measure of which the patients follow the instructions of the therapeutic treatments [12], and it is one of the most difficult aspects to manage in the patients affected by CKD. Pharmacological non-adherence can be dangerous for the patients' health and could result 


\section{Nursing \& Healthcare International Journal}

in worsening the progression of the CKD to the final stage of the disease [13].

Therapeutic education in chronic diseases is strictly related to therapeutic adherence. For this reason, this approach is already in use for the management of type 1 diabetes in the management of insulin therapy in the community [14], after renal transplant to ensure immunosuppressive administration [15] and in the management of the heart failure to improve therapeutic adherence [16]. World Health Organization (WHO) estimates that only half of the people affected by chronic diseases self-administer pharmacological therapy as prescribed [17]. Levels of non-adherence are variable and are reported in several scientific studies: an article from 2015, for example, highlights that $26-28 \%$ of patients affected by CKD are non-adherent to therapeutic prescriptions [18].

Factors that influence therapeutic non-adherence are aspects of the relationship between doctor and patient (including the ability to offer effective communication), time available for the visits [19], opportunity to have a continuous relationship with the same doctor $[20,21]$, patient's characteristics (demographics, self-management, empowerment, locus of control) and the type of pathology (medical history, seriousness of the disease, symptoms, co-morbidities).

Self-management, together with expectations, seem to have a main role in determining a behavior and therefore seem powerful predictors of behaviors relevant to the health. Own beliefs about self-management consist of judgement about own capabilities to organize and execute actions while expectations refer to judgements about the possible consequences that those actions may produce [22].

Another important factor is the patient's empowerment, defined as education process aiming to help the patient to develop knowledge, abilities, attitudes and self-awareness needed to become responsible of the decisions about their own health [23]. Amongst factors related to empowerment we can find locus of control, which consists in the perception of control that the subject has towards a situation's outcome: an internal locus of control would tend to make the subject to perceive the events as controllable and therefore to facilitate adaptive solutions to the disease to facilitate adaptive solutions to the while an external locus of control would facilitate negative coping mechanisms [24]. Moreover the intrinsic characteristics of the treatment and the patient's perceptions are strongly linked to adherence, amongst those: treatment complexity [25], duration [26], side effects, how quick the treatment start working, patient's level of knowledge, expectations, fear of substance abuse and over prescription [27]. Despite the absence of a profile of the compliant patient, the above factors can have different impacts on different patients, to confirm the central role of the patient's compliance to the pharmacologic therapy. Among the variables related to non-adherence to the therapy, associated to the patient, it is to mention the difficulties of the patient to recognize their own condition of being patient and the need for treatment [28], therefore it is to pay attention to the patient's subjective perception of the disease.

The disease's perception becomes important if it is considered that the behavioral response to threats to health are mediated by the patient's intrinsic theories about the disease [29].

The assessment of the disease's perception is a "frame" where are situated variables as correct communication of the medical issue, planning of the care, prevention and education appropriate for the subject and a prognostic evaluation able to merge prevention and predictive situation [30].

It's easy to come across situations where the disease is partially denied, or where the seriousness or chronicity are denied, resulting in a partial adherence or full adherence without believing in it. The understanding of the complexity of the disease, and of the treatment nonetheless, together with the understanding of the benefits in medium to long term related to the specific treatment represents the best prompt to the patient and therefore improves the compliance [31]. There is evidence that interventions aimed to improve compliance have a bigger influence in the population health compared to any other improved specific treatments [32].

The current model, used to assess barriers to compliance, is focused on the interactions between the patient, healthcare professional and the health system. Unfortunately, most of the researches, aimed to improve empowerment and adherence through psycho-educative tools, are not focused on ADPKD.

\section{Materials and Methods}

Quasi experiment study, pre-post test, longitudinal, multi-centric, in patients affected by ADPKD with chronic kidney disease (CKD). A sample of 50 patients will receive an intervention of therapeutic education on compliance with pharmacological therapy, diet and lifestyle. 


\section{Nursing \& Healthcare International Journal}

\section{Settings and Participants}

A sample of 50 patients affected by ADPKD, will be recruited in the ADPKD clinic at San Raffaele Hospital in Milan and in the Nephrology clinic at Federico II University Hospital in Naples, over a period of 18 months. Inclusion criteria are: 1) age $>18$ years old; 2) diagnosis of CKD as per eGFR CKD-EPI; [33] 3) Pharmacological treatment includes Tolvaptan; 4) consensus signed by patient.

Exclusion criteria are: 1) patients receiving dialysis; 2) patients that received renal transplant; 3) patients with mental health issues; 4) patients with cognitive impairment.

\section{Sampling}

A consecutive non-probability sampling method will be used to recruit 50 subjects that will attend the ADPKD clinic in San Raffaele Hospital in Milan and the Nephrology clinic at Federico II University Hospital in Naples. The patients will receive planned interventions of therapeutic education.

\section{Objectives}

The primary objective of the study is to assess the effects of therapeutic education in patients affected by ADPKD and CKD, on level of compliance to pharmacological treatments, diet and lifestyle behaviors. The reason to choose this objectives is based on the concept that education can have a positive effect on the level and quality of the patient's knowledge about the disease and pharmacologic therapy (side effects, risks, benefits, modalities of administration), on the diet and lifestyles that are healthy, with a possible improvement of the compliance and empowerment.

The secondary objectives are to assess the impact of therapeutic education on the management of clinical conditions, the perception of the disease, the management of complications, the ability to self-care, the quality of life and patient engagement, locus of control and the multidisciplinary approach to the care. These objectives have been chosen due to the fact that these are dependent variables greatly influenced by therapeutic education.

\section{Research Questions}

1. Does the use of therapeutic education in patients with ADPKD associated with CKD increase compliance with drug therapy, dietary prescription, attitudinal and health lifestyles, empowerment, control of clinical conditions and the perception of the disease?

2. Can therapeutic education be useful to promote Tolvaptan tolerance by providing the patient with strategies to manage polyuria and of proper hydration?

3. Does the use of therapeutic education reduce complications by increasing self-care skills, improving quality of life, patient involvement, control of the locus, and improving multidisciplinary care?

\section{Data Collection}

The study consist in two visit: Visit 1 (V1) and Visit 2 (V2). V1 represent the recruitment phase and the baseline record (pre-test). A nurse taught on the experimental protocol, called data collector, will apply inclusion and exclusion criteria to assess eligibility of every patient.

To the patients whom meet the inclusion criteria will be offered the opportunity to join the study by a nurse and a psychologist, providing the patient with details of the study and assessing the attitude and the availability of the patient to participate in the study. The patients that will accept to join the study will be recruited. To the recruited patients will be explained: a) the form for patient's sensitive data treatment, to guarantee data safety and privacy agreement with signature; b) information form of the study for the patient; c) informed agreement module of participation to the study with signature; d) letter for the General Practitioner.

During the first visit socio-demographics, details will be gathered by the use of the Case Report Form (CRF), and every patient will complete different questionnaires:

Patient Assessment of Chronic Illness Care (PACIC), International Physical Activity Questionnaire (IPAQ), Simplified Medication Adherence Questionnaire (SMAQ), Multidimensional Health Locus of Control Scale (MHLC), Illness Perception Questionnaire - Revised Brief (BIPQ), Patient Activation Measurement (PAM) and the Kidney Disease Quality of Life - Short Form (KDQOL-SF).

V2 represent the post-test visit, in which the nurse called outcome assessor, will gather the scores assigned by the patient to the questionnaires PACIC, IPAQ, SMAQ, MHLC, BIPQ, PAM and KDQOL-SF after three months of intervention with therapeutic education of the patients. The visit framework is shown in Table 1. 


\section{Nursing \& Healthcare International Journal}

\begin{tabular}{|c|c|c|c|}
\hline Visits & V1 (baseline) & V2 & Tool \\
\hline Time & Day 1 & Month 3 & \\
\hline Procedure & & & \\
\hline Inclusione/exclusion criteria & $\mathrm{x}$ & & \\
\hline Eligible patients are offered to join the study & $\mathrm{X}$ & & \\
\hline Information to the patient - privacy & $\mathrm{x}$ & & \\
\hline Informed consensus - sensitive data treatment & & & \\
\hline Information's for the patient - participation to the study & $\mathrm{X}$ & & \\
\hline Informed consensus - participation to the study & $\mathrm{X}$ & & \\
\hline Letter to the General practitioner & $\mathrm{X}$ & & \\
\hline Socio-demographics and clinical data & $\mathrm{X}$ & & $\mathrm{CRF}$ \\
\hline Chronic Illness Care & $\mathrm{X}$ & $\mathrm{X}$ & PACIC \\
\hline Physical Activity & $\mathrm{x}$ & $\mathrm{X}$ & IPAQ \\
\hline Medication adherence & $\mathrm{x}$ & & SMAQ \\
\hline Health Locus of Control & $\mathrm{x}$ & $\mathrm{x}$ & MHLC \\
\hline Illness Perception & $\mathrm{x}$ & $\mathrm{X}$ & $\mathrm{BIPQ}$ \\
\hline Patient Activation & $\mathrm{x}$ & $\mathrm{X}$ & PAM \\
\hline Quality of Life & $\mathrm{x}$ & $\mathrm{X}$ & KDQOL-SF \\
\hline Adverse reactions & & $\mathrm{X}$ & Frequency (\%) \\
\hline Withdrawals & $\mathrm{X}$ & Frequency (\%) \\
\hline
\end{tabular}

Table 1: Framework of the visits, assessment and tools.

\section{Intervention}

Therapeutic education will be performed by a nurse in the ADPKD clinic at San Raffaele Hospital in Milan and in the Nephrology clinic of the Federico II University Hospital in Naples. The nurse will use standardized educational procedures to educate the patient on the different modalities of medication's administration, supported by written advices and communication supports. A leaflet that indicated the food with high level of sodium, the maximum level of sodium allowed per day $(5 \mathrm{~g})$ and the advice of completing moderate physical activity will provided.

The nurse will explain the risks and benefits related to provided prescriptions, will prompt the patient to ask any question that it is felt necessary and will ask the patient to repeat all the essential information that have been provided.

Therapeutic education will be performed in one single visit of about 45 minutes per patient.

\section{Ethical Considerations}

This study will be completed following the principles of the Good Clinical Practice dell'ICH Harmonized Tripartite Guidelines for Good Clinical Practice 1196
Directive 91/507/EECe del DM 15.07.1997, the Helsinki declaration and the national laws regarding clinical trials.

\section{Measurements}

1) Case report form (CRF), to gather the following values: age, gender, civil status, level of education, available resources, psychiatric conditions, medical history, presence/absence of symptoms, number of medications prescribed, presence of side effects from the treatment, costs of the pharmacological therapy, level of information on the disease, drugs and diet, quality of doctor/patient relationship, length of access to the clinic.

2) Patient Assessment of Chronic Illness Care (PACIC) [34]. This tool will be used to assess the care pathways for chronic diseases. It has 26 items that relates to the care received in the previous 6 months and allows to divide the scores in 5 sub-groups: patient's engagement, support in the decision making process, goals setting, problem-solving/counselling and follow up.

3) International Physical Activity Questionnaire (IPAQ) [35]. Questionnaire about daily physical activity, 31 items on different levels of physical activity in the last 7 days: low, moderate, high. 


\section{Nursing \& Healthcare International Journal}

4) Simplified medication adherence questionnaire (SMAQ) [36]. Validated tool for patients that received renal transplant, 6 items about the habits of medications consumption, related to an improved control of the pharmacological therapy and possible reduction of side effects. We used this simple questionnaire es widely used in chronic disease and we wanted to considerer Tolvaptan as a chronic drugs. A higher sensitivity is advantageous in a tool such as this, since it provides a greater power of detection of non-compliant subjects and thus leads to better clinical follow-up.

5) Multidimensional Health Locus of Control Scale (MHLC) [37]. Made of three sub-groups of 6 items each of two equivalent forms. Every form contains 18 items. Three sub-groups are Internal Locus of Control (Internal Health Locus of Control - IHLC), External Locus of Control (Powerful Others Externality - PHLC) and Casual Locus of Control (Chance Health Locus of Control - CHLC). Likert like scales with 6 points, can go from strongly disagree to strongly agree.

6) Illness Perception Questionnaire - Revised Brief (BIPQ) [38]. Questionnaire with good validity and reliability, it's used to assess the patient's point of view about the disease. It gives information about: perception of causes and consequences, duration, perception on personal control and treatment, emotional response, disease identity. High scores indicate a threatening perception of the disease.

7) Patient Activation Measurement (PAM) [39]. 13 items, valid and statistically reliable. Every item has 5 answers from 1 to 5: 1) "strongly disagree"; 2) "disagree"; 3) "agree"; 4) "strongly agree"; 5) "don't know". The aim of the tool Is to complete an assessment of the level of proactivity of the patient in the management of the disease by assessing the patient's knowledge of the disease and the perception of the level of skills and self-management. The patient can be allocated to 4 different levels: level 1 (Important role in the management of the disease); level 2 (Patient is self-conscious in order to manage the disease); level 3 (Patient takes actions); level 4 (Patient is able to respond to stress in order to manage the disease). It is a useful tool in the evaluation of interventions aimed to improve the involvement of the patient in the health status management and to train clinicians to modify their communication style based on the level of patient's engagement.

8) Kidney Disease Quality of Life - Short Form (KDQOLSF) [40]. Questionnaire to asses perception of quality of life in patients with renal disease, 87 items. The version used is made of items of a generic questionnaire about quality of life (SF-36) and other specific items related to the renal disease. The first part is made of 36 items divided in 8 subgroups (Physical function, physical role, pain, health status, emotional wellbeing, role of emotional status, social function, energy). These items can be grouped in two different indexes, the Physical Health Index (PHI) and the Mental Health Index (MHI). The second part of the test is specific to the renal disease and includes 42 items divided in 9 subgroups (Symptoms, effects of the disease, gravity of the disease, Jon, cognitive function, social interactions, sexual function, sleep, social support). The third part is made of 6 items related to the levels of satisfaction of the service: this section has not been used because it is specific to patients on dialysis therapy. The fourth part has 3 items that gathers socio-demographics data. The score is assessed by considering each sub-group different score; to high scores correspond a better health status of the patient. The score goes from a minimum of 0 to a maximum of 100, 50 are the mean score. The reliability and validity of the tool are supported by several studies and preliminary studies shows that is also a good tool as a predictor for hospitalization and mortality [41].

\section{Statistical Analysis}

The factor variables are expressed in frequencies and percentages while continuous variables are expressed in means and standard deviations for normally distributed variables and medians and quartiles for the measurement of the variables not normally distributed. To compare the characteristics of the patients and between Visit 1 and Visit 2 the Student t-test will be utilized. For the pre-post variables correlations the Pearson correlation index will be used. Statistical significance will be considered if $\mathrm{p}<.05$ (two-tailed significance). Statistical significance will be completed using SPSS тм (IBM Statistics for Windows v.20).

\section{Conclusions}

In ADPKD it is observed that an increased number of patients have an increased number of medications and it is essential to utilize tools and methods to assess and improve pharmacological therapy compliance. Moreover, a healthy lifestyle can delay the worsening of the symptoms and the disease's progression, therefore the introduction of a structured therapeutic education could represent a valid strategy to improve patient's compliance to therapies. Poor compliance represents the main cause of pharmacological inefficiency it is associated with an 


\section{Nursing \& Healthcare International Journal}

increased number of interventions by the careers, comorbidity and mortality, resulting in damage to the patient, health system and society. The advantages brought by this study could be: a) increased patient's knowledge of the disease, therapies and positive behaviors in order to promote an autonomous management of the disease and benefits for the physical and psychological wellbeing; b) modify wrong behaviors in order to obtain a balance between patient's needs and the disease; c) improve compliance to therapies resulting in a more effective treatment and a better quality of life; d) obtain and maintain the skills and competencies to live in the best way possible the daily relationship with the disease.

This study aims to explore new rehabilitation and assistance measures aimed at managing deficits, consequential functional disabilities caused by the disease for a better reinsertion of the patient in the family, social and work environment, increasing the quality of his life.

For future studies it would be useful to validate a specific questionnaire to measure Tolvaptan adhesion

\section{References}

1. Neumann HP, Jilg C, Bacher J, Nabulsi Z, Malinoc A, et al. (2013) A Epidemiology of autosomal-dominant polycystic kidney disease: an in-depth clinical study for south-western Germany. Nephrol Dial Transplant 28(6): 1472-1487.

2. Chapman AB (2008) Approaches to testing new treatments in autosomal dominant polycystic kidney disease: insights from the CRISP and HALT-PKD studies. Clin J Am Soc Nephrol 3(4): 1197-1204.

3. Torres VE, Abebe KZ, Schrier RW, Perrone RP, Chapman AB, et al. (2017) Dietary salt restriction is beneficial to the management of Autosomal Dominant Polycystic Kidney Disease, Kidney Int 91(2): 493-500.

4. Tran WC, Huynh D, Chan T, Chesla CA, Park M (2017) Understanding barriers to medication, dietary, and lifestyle treatments prescribed in polycystic Kidney disease. BMC Nephrology 18(1): 214.

5. Chapman AB, Devuyst O, Eckardt KU, Gansevoort RT, Harris T, et al. (2015) Autosomal Dominant Polycystic Kidney Disease (ADPKD): Executive Summary from a Kidney Disease: Improving Global Outcomes (KDIGO) Controversies Conference 88(1): 17-27.
6. Esposito P, La Milia V, Altobelli C, Cerutti R, Manunta P, et al. (2018) Practical approach to patient therapy affected by Autosomal Dominant Autosomic Polycystic Kidney Disease. G Ital Nefrol 35(4).

7. Beghelli A, Ferraresi A, Manfredini M, (2015) Educazione terapeutica Metodologia e applicazioni, Carocci Faber.

8. Epping Jordan JE, Pruitt SD, Bengoa R, Wagner EH, (2004) Improving the quality of health care for chronic conditions. Qual Saf Health Care 13(4): 299305.

9. Wagner EH, Davis C, Schaefer J, Von Korff M, Austin B (1999) A survey of leading chronic disease management programs: are they consistent with the literature? Manag Care Q 7(3): 56-66.

10. D Ivernois JF, Gagnayre R, Edizione Italiana MG Albano, Sasso L (2009) Educare il paziente. Un approccio pedagogico, McGraw-Hill, Milano.

11. Weil AR (2016) The Patient Engagement Imperative, Health Aff (Millwood) 35(4): 563.

12. McDonald HP, Garg AX, Haynes RB (2002) Interventi per migliorare l'aderenza dei pazienti alle prescrizioni di farmaci: revisione scientifica. JAMA 288: 2868-2879.

13. Sontakke S, Budania R, Bajait C, Jaiswal K, Pimpalkhute S (2015) Evaluation of adherence to therapy in patients of chronic kidney disease. Indian J Pharmacol 47(6): 668-671.

14. Cartabellotta A, Manicardi V, Coscelli C (2016) Guidelines for the treatment of type 2 diabetes in adults. Evidence 8(12): e1000159.

15. Dell Angelo Simona (2017) Empowerment: a strategy for the therapeutic education of the patient undergoing a living donor kidney transplant. Bachelor thesis, University of Applied Sciences of Italian Switzerland (SUPSI).

16. Dunbar SB, Clark PC, Reilly CM, Gary RA, Smith A, et al. (2013) A trial of family partnership and education interventions in heart failure. J Card Fail 19(12): 829841.

17. World Health Organization (2015) Adherence to longterm therapies: action trials. 


\section{Nursing \& Healthcare International Journal}

18. Magacho EJ, Ribeiro LC, Chaoubah A, Bastos MG (2011) Adherence to drug therapy in renal disease. Braz J Med Biol Res 44: 258-262.

19. Kyngas H, Rissanen M (2001) Support as a crucial predictor of good compliance of adolescents with a chronic disease, 10(6): 767-774.

20. Pendley JS, Kasmen LJ, Miller DL, Donze J, Swenson C, et al. (2002) Peer and family support in children and adolescents with type 1 diabetes, J Pediatr Psychol 27(5): 429-438.

21. Horne R, Weinman J (1999) Patients' beliefs about prescribed medicines and their role in adherence to treatment in chronic physical illness. J Psychosom Res 47(6): 555-567.

22. Bandura A (2000) Self-efficacy: The foundation of agency. In: Perring WJ, et al. (Eds), Control of human behaviour, mental processes and consciousness Mahwak, NJ: Erlbaum pp: 17-33.

23. Feste C, Anderson RM (1995) Empowerment: from philosophy to practice. Patient Educ Couns 26(1-3): 139-144.

24. Crisson JE, Kafee FJ (1988) The relationship of locus of control to pain coping strategies and psychological distress in chronic pain patients. Pain 35(2): 147-154.

25. Griffith R (2004) Living wills, duty of care and the right to treatment. Br J Community Nurs 488-491.

26. Sherbourne CD, Hays RD, Ordway L, DiMatteo MR, Kravitz RL (1992) Antecedents of adherence to medical recommendations: results from the Medical Outcomes Study. J Behav Med 15(5): 447-468.

27. Logan AG, Milne BJ, Achber C, Campbell WP, Haynes RB (1979) Work-site treatment of hypertension by specially trained nurses. A controlled trial. Lancet 2(8153): 1175-1178.

28. Meichenbaum D, Turk DC (1987) Facilitating treatment adherence: A practitioner's guidebook. New York.

29. Weinman J, Petrie KJ (1997) Illness perceptions: a new paradigm for psychosomatics? J Psychosom Res 42(2): 113-116.

30. Trovato GM, Catalano D, Martines GF, Spadaro D, Garufi G, et al. (2016) Perception of Disease, IPQ-r: a useful paradigm in chronic diseases NPM Recent advances in medicine 97(3): 129-133.
31. Miselli V (2011) Il problema dell'adesione alla terapia in una malattia cronica come il diabete. G It Diabetol Metab 31: 121-124.

32. Haynes RB, Montague $\mathrm{P}$, Oliver $\mathrm{T}$, McKibbon KA, Brouwers MC, et al. (2000) Interventions for helping patients to follow prescriptions for medications. Cochrane Database Syst Rev (2): CD000011.

33. Levey AS, Stevens LA, Schmid CH, Zhang YL, Feldman $\mathrm{HI}$, et al. (2009) A New Equation to Estimate Glomerular Filtration Rate. Ann Intern Med 150(9): 604-612.

34. Glasgow RE, Wagner E, Schaefer J, Mahoney L, Reid, et al. (2005) Development and validation of the Patient Assessment of Chronic Illness Care (PACIC). Med Care 43(5): 436-444.

35. Mannocci A, Di Thiene D, Del Cimmuto A, Masala D, Boccia A, et al. (2010) International Physical Activity Questionnaire: validation and assessment in an Italian sample. Ital J Public Health 7(4): 369-376.

36. Knobel H, Alonso J, Casado JL, Collazos J, González J, et al. (2002) GEEMA Study Group. Validation of a simplified medication adherence questionnaire in a large cohort of HIV-infected patients: the GEEMA Study. AIDS 16(4): 605-613.

37. Wallston, Wallston, De Vellis R (1978) Development of the Multidimensional Health Locus of Control (MHLC) Scales. Health Educ Monogr 6 (1): 160-170.

38. Broadbent E, Petrie KJ, Main J, Weinman J (2006) The brief illness Perception Questionnaire. J Psychoso Res 60(6): 631-637.

39. Graffigna G, Barello S, Bonanomi A, Lozza E, Hibbard J (2015) Measuring patient activation in Italy: Translation, adaptation and validation of the Italian version of the patient activation measure 13 (PAM13I). BMC Med Inform Decis Mak 15: 109.

40. Klersy C, Callegari A, Giorgi I, Sepe V, Politi P, (2007) Italian translation, cultural adaptation and validation of KDQOL-SF, version 1.3, in patients with severe renal failure. J Nephrol 20(1): 43-51.

41. Mapes DL, Lopes AA, Satayathum S, McCullough KP, Goodkin DA, et al. (2003) Health-related quality of life as a predictor of mortality and hospitalization: the Dialysis Outcomes and Practice Patterns Study (DOPPS). Kidney Int 64(1): 339-349. 